\title{
Association of DNA Polymorphisms in the Synaptic Vesicular Amine Transporter Gene (SLCI8A2) with Alcohol and Nicotine Dependence
}

\author{
Sibylle G Schwab*,1,2,3,4,9, Petra E Franke ${ }^{4,9}$, Barbara Hoefgen ${ }^{4}$, Vera Guttenthaler ${ }^{4}$, Dirk Lichtermann ${ }^{4,5}$, \\ Matyas Trixler, Michael Knapp ${ }^{7}$, Wolfgang Maier ${ }^{4}$ and Dieter B Wildenauer ${ }^{1,8}$ \\ 'School of Psychiatry and Clinical Neurosciences, University of Western Australia, Perth, WA, Australia; ${ }^{2}$ Centre for Medical Research, University of \\ Western Australia, Perth, WA, Australia; ${ }^{3}$ Western Australian Institute for Medical Research, Nedlands, WA, Australia; ${ }^{4}$ Department of Psychiatry, \\ University of Bonn, Bonn, Germany; ${ }^{5}$ Methadon Maintenance Clinic 'Café-Ersatz', Bonn, Germany; ${ }^{6}$ Department of Psychiatry, University of Pecs, \\ Pecs, Hungary; ${ }^{7}$ Institute for Medical Biometry, Informatics and Epidemiology, University of Bonn, Bonn, Germany; ${ }^{8}$ Centre for Clinical Research in \\ Neuropsychiatry, University of Western Australia, Mt. Claremont, WA, Australia
}

\begin{abstract}
The brain synaptic vesicular amine transporter SLCA I8A2 is a key component for the uptake of monoamines like dopamine or serotonin into vesicles. We have analyzed seven DNA polymorphisms located in the genomic region of SLCI 8A2 for association with alcohol- and nicotine dependence, using a family-based design. Our sample comprised I 3 I families with alcohol-dependent offspring and 96 families with at least one nicotine-dependent offspring. For the alcohol-dependent sample, we found statistical significant association for two single markers (rs363387, $P=0.03 ; r s 363333, P=0.0066$ ) as well as for several haplotypes (minimal $P=0.0038$ ). When the sample with alcohol dependence was stratified according to gender, we observed increased association for the male subgroup (rs363387, $P=0.00 \mathrm{II}$ ). None of the markers showed association in the sample of families with nicotine dependence. However, analysis of a combined sample of alcohol and nicotine-dependent families resulted in single markers as well as several haplotypes showing statistical significant association with substance dependence (minimal $P=0.0044$ ). We conclude that DNA polymorphisms located in SLCI8A2 might contribute to the development of substance dependence.

Neuropsychopharmacology (2005) 30, 2263-2268. doi: I 0. I038/sj.npp. I 300809; published online 29 June 2005
\end{abstract}

Keywords: synaptic vesicular amine transporter; alcohol dependence; nicotine dependence; association; transmission disequilibrium test; substance dependence

\section{INTRODUCTION}

The contribution of genetic factors to alcohol and nicotine dependence is well established by family, twin, and adoption studies (Dinwiddie and Reich, 1993; Sigvardsson et al, 1996; Sullivan and Kendler, 1999). For nicotine dependence, estimates indicate that genetic contribution to smoking initiation in twins accounts for $60 \%$ of the variance, while heritability might account for $70 \%$ of the variance in persistent smoking and nicotine dependence (Sullivan and Kendler, 1999). For alcoholism, heritability estimates range from 50 to $60 \%$, with heritability expected to be particularly high in a subgroup of male alcoholics,

\footnotetext{
*Correspondence: Dr SG Schwab, Western Australian Institute for Medical Research, Sir Charles Gairdner Hospital, B-Block, Ground Floor, Hospital Ave., Nedlands, 6009 WA, Australia, Tel: +61 89346 27II, Fax: +61 89346 |8|8, E-mail: sschwab@cyllene.uwa.edu.au ${ }^{9}$ These two authors contributed equally.

Received 19 January 2005; revised 4 April 2005; accepted 22 May 2005 Online publication: 26 May 2005 at http://www.acnp.org/citations/ Npp052605050044/default.pdf
}

characterized by antisocial behavior, aggression, impulsivity, early onset of the disorder, and multisubstance abuse (Sigvardsson et al, 1996).

Individuals who regularly smoke are also at high risk for becoming alcohol dependent. Twin studies examining the relationship between smoking and alcohol use have demonstrated common and specific genetic factors for both. In addition, a strong association between heavy drinking and smoking has been shown (Madden et al, 1995). Consequently, it has been suggested that a common genetic vulnerability to nicotine and alcohol dependence in men might exist (True et al, 1999).

In unraveling the genetic background of substance dependence genes involved in neurotransmitter systems have been a major focus. The brain reward circuits include dopamine projections from the ventral tegmental area and substantia nigra to the nucleus accumbens and striatum, as well as glutamate inputs from the prefrontal cortex, amygdala, and hippocampus. Drug sensitization-related changes have been described in many neurotransmitter systems that are integral to the function of the reward 
system, that is, dopamine, serotonin, norepinephrine, acetylcholine, opioid, and GABA systems (Robinson and Berridge, 2003). Recently, a report by Heinz et al (2004) described reduced dopamine D2 receptor availability in detoxified alcoholics. These authors suggested that dopaminergic dysfunction in the ventral striatum may attribute incentive salience to alcohol-associated stimuli.

One of the proteins necessary for a proper functioning of monoaminergic neurons is the brain vesicular amine transporter (SLC18A2, VMAT2). This transporter uses energy from vesicular proton gradients to accumulate monoamine neurotransmitters from the neuronal cytoplasm into synaptic vesicles (Johnson, 1988; Henry et al, 1994). The selectivity with which monoaminergic neurons package dopamine, serotonin, norepinephrine, or histamine into synaptic vesicles by SLC18A2 is derived from the specificity of the corresponding plasma membrane transporters. Predominant substrates for SLC18A2 are, among others, serotonin and dopamine; therefore, making it an ideal candidate gene for analysis of association with substance dependence. Moreover, Straub et al (1999) published a genome scan on nicotine addiction, with chromosome 10q26, the chromosomal location of SLC18A2, being one of the putatively linked regions. A follow-up of this study, using a computational approach, which allows searching for epistatic loci, identified SLC18A2 as a prime candidate in substance dependence (Sullivan et al, 2004).

Based on the pivotal neurobiological role as well as on the location in a region putatively linked with nicotine addiction, we have analyzed seven DNA polymorphisms in the genomic region of SLC18A2 for association with nicotine and alcohol dependence. We have used a familybased approach in order to avoid population stratification.

\section{MATERIALS AND METHODS}

\section{Family Samples}

Alcohol-dependent individuals fulfilling DSM-IV criteria were recruited from consecutive voluntary admissions for detoxification of alcohol at the Department of Psychiatry, Universities of Bonn, Germany and Pecs, Hungary. Patients with other types of illegal substance abuse were excluded from recruitment. A total of 131 alcohol-dependent patients with both biological parents gave informed consent for participation in the study and for providing DNA for genetic research on addiction. Approximately $70 \%$ of the alcohol-dependent patients were current smokers. A subset of the families with alcoholism have been included in a dopamine transporter 1 (SLC6A3) association study (Franke et al, 1999).

Nicotine-dependent individuals have been recruited by staff members of the Department of Psychiatry, University of Bonn, by advertisement at the University campus indicating the scientific purpose of the study. Only individuals with no history of major psychiatric disorder were included. The majority of the probands consisted of students. All consenting smokers were screened for smoking habits using the Fagerstrom questionnaire (Heatherton et al, 1991) and underwent a semistructured diagnostic interview procedure for major psychiatric disorders including substance abuse. Probands fulfilling DSM-IV criteria for any kind of other substance dependence than nicotine were excluded. Probands from the nicotine dependence group were habitual smokers with more than 20 cigarettes per day for at least 1 year. Altogether 96 families have been recruited comprising one family with five nicotine-dependent offspring, one family with four, six families with three, 21 families with two, and 67 families with one nicotinedependent offspring. For all 96 families, both biological parents were available for genotyping. Written informed consent was obtained from all study participants.

Ethical approval has been obtained from the local committees of the University of Bonn and University of Pecs.

\section{Genotyping}

DNA was isolated either from whole blood or permanent cell cultures derived from Epstein-Barr virus transformed lymphocytes using a Qiagen Blood and Cell Culture Kit (Qiagen, Hilden, Germany). The present analysis comprised seven SNPs spanning $34.82 \mathrm{~kb}$ of the genomic region of the SLC18A2 gene on chromosome 10q26.11 (Table 1). SNPs were selected according to intermarker distance and their minor allele frequency. Priority was given to SNPs located in exonic regions. Intermarker linkage disequilibrium $\left(D^{\prime}\right.$ and $r^{2}$ ) calculated using FAMHAP and both family samples indicates that markers in the $3^{\prime}$ and $5^{\prime}$ region of the gene are in moderate linkage disequilibrium (Table 2). This is also supported by data available from the HapMap project (http://www.cshl.hapmap.org/). For all SNPs, Taqman SNP genotyping assays on demand were available from Applied Biosystems (Applied Biosystems, Foster City, CA, USA). PCR was performed using $12.5 \mathrm{ng}$ of DNA according to the protocol of Applied Biosystems. Fluorescence was measured on completion of the PCR by means of a Tecan Ultra 384 reader (Tecan, Crailsheim, Germany). Excitation- and emission wavelengths for the fam labeled probes were 485 and $535 \mathrm{~nm}$ and for the vic labeled probes 535 and $590 \mathrm{~nm}$, respectively.

\section{Statistical Methods}

Version 12 of the program FAMHAP (Becker and Knapp, 2004a) was used for estimating haplotype frequencies and

Table I Location and Characterization of SNPs in the SLCI8A2 Gene Used in the Analysis

\begin{tabular}{lclc}
\hline & Distance to next SNP & Location & Polymorphism $^{\text {a }}$ \\
\hline rs363387 & $515 \mathrm{bp}$ & Exon 2 & $\mathrm{T} / \mathrm{G}^{\mathrm{b}}$ \\
rs363390 & $16 \mathrm{bp}$ & Intron 2 & $\mathrm{G} / \mathrm{C}$ \\
rs363333 & $10851 \mathrm{bp}$ & Intron 2 & $\mathrm{T} / \mathrm{C}$ \\
rs363343 & $4176 \mathrm{bp}$ & Intron 6 & $\mathrm{A} / \mathrm{C}$ \\
rs929493 & $51 \mathrm{bp}$ & Intron 9 & $\mathrm{T} / \mathrm{C}$ \\
rs1890404 & $1921 \mathrm{bp}$ & Intron 9 & $\mathrm{C} / \mathrm{T}$ \\
rs363236 & & 3' downstream & $\mathrm{T} / \mathrm{C}$ \\
\hline
\end{tabular}

${ }^{a}$ First allele is the common allele.

${ }^{b}$ Transversion results in a synonymous change of threonine at amino-acid position 68 
Table 2 Intermarker LD as Calculated by FAMHAP Using Alcohol- and Nicotine-Dependent Families

\begin{tabular}{|c|c|c|c|c|c|c|c|}
\hline & rs363387 & rs363390 & rs363333 & Rs363343 & rs929403 & rsI890404 & rs363236 \\
\hline rs363387 & & 0.7606 & I & 0.0330 & 0.0381 & 0.0486 & 0.2847 \\
\hline rs363390 & 0.0073 & & 0.8485 & 0.4508 & 0.4318 & 0.4134 & 0.0428 \\
\hline rs363333 & 0.6134 & 0.0148 & & 0.2411 & 0.0011 & 0.1579 & 0.1632 \\
\hline rs363343 & 0.0003 & 0.1403 & 0.0008 & & 0.9794 & I & 0.0777 \\
\hline rs929403 & 0.0004 & 0.1239 & 0.000001 & 0.9232 & & I & 0.0827 \\
\hline rs363236 & 0.0311 & 0.0010 & 0.0167 & 0.0049 & 0.0058 & 0.0079 & \\
\hline
\end{tabular}

Above the diagonal are values for $D^{\prime}$, below the diagonal are values for $r^{2}$.

for testing for association of single markers and haplotypes. FAMHAP provides a haplotype TDT-like test, which is valid as a test of association even in the presence of linkage and in case that the families contain more than one single affected child (Knapp and Becker, 2003). Simulations are performed to assess an uncorrected $P$-value for a specific marker combination. In case that more than a single marker combination is tested for its association with the disease, FAMHAP also enables the calculation of a global $P$-value being corrected for multiple testing (Becker and Knapp, 2004b).

Intermarker linkage disequilibrium was calculated using FAMHAP and the frequencies of the nontransmitted alleles (pseudo-controls) in the combined family sample.

Power calculations have been performed to assess the potential resolution of the alcohol-dependent family sample as described by Knapp (1999).

\section{RESULTS}

We have studied 131 nuclear families with a single alcoholdependent offspring and 96 families with at least one offspring affected with nicotine dependence for association with SNPs located in the genomic region of the vesicular monoamine transporter 2 (SLC18A2) on chromosome 10q26.11. Family-based association analysis was performed independently for the sample of families with alcohol dependence and for the family sample with nicotine dependence. Since as many as $80 \%$ of alcohol-dependent individuals also have a history of cigarette abuse and the evidence in the literature suggests that those susceptible to dependence or addiction tend to be multiple substance abusers (True et al, 1999; Bierut et al, 2004), we have also carried out an association analysis for the combined sample of alcohol- and nicotine-dependent families.

All markers were in Hardy-Weinberg equilibrium for all samples. Single marker analysis revealed statistical significant association for markers rs363387 and rs363333 with $P=0.0325$ and 0.006 , respectively in the family sample with alcoholism. In the sample with nicotine-dependent families, overtransmission for the same alleles of these markers was observed, but no statistical significant association was obtained (Table 3). Analysis of the combined sample revealed statistical significant $P$-values for the two markers rs363387 $(P=0.0190)$ and $r 3363333(P=0.0078)$ (Table 3$)$, suggesting a contribution of the nicotine-dependent families to the association result.

Since there is substantial evidence for sex-dependent differences in alcoholism, with specific alcoholism subtypes more represented in male than in female subjects (Cloninger et al, 1988), we analyzed the alcohol dependent families according to the gender of the affected offspring. Of the 131 families with alcohol dependence, 115 had a male offspring. $P$-values for markers rs363387 and rs363333 in this subsample were slightly smaller $(P=0.0011$ and 0.0035 , respectively) than those observed in the whole sample (Table 4). In addition, marker rs 363390 , which is $515 \mathrm{bp}$ downstream of marker rs363387, reached statistical significance just below the 5\% level $(P=0.0402)$. In the subsample with female offspring, no statistical significant $P$-value was observed. Since the female group was quite small $(n=16)$, this subsample was not analyzed further for association of SLC18A2 DNA polymorphisms with alcoholism.

We performed haplotype analysis in order to increase the information of the single SNP markers in our analysis. All possible single-, two- and three-marker haplotype combinations were computed using FAMHAP. Simulated overall significance, corrected for multiple testing was $P=0.045$ for the sample of 131 alcohol-dependent families. A two-locus haplotype comprising markers rs363387 and rs363333 revealed the smallest uncorrected $P$-value of 0.0038 (Table 5). Furthermore, most marker combinations that included markers rs363387 and rs363333 produced $P$-values less than 0.05 . In contrast, haplotypes of the combined sample (alcohol and nicotine-dependent families) produced statistical significant $P$-values when marker rs363236 was included (Table 5). This marker is located approximately $35 \mathrm{~kb}$ downstream of marker rs363387. For the combined sample, simulated overall significance was $P=0.0477$. Again, no statistical significant $P$-values were obtained for the sample of 96 families with nicotine dependence.

When families with alcohol dependence were stratified according to gender, no significant $P$-values were obtained for the families with female offspring. In contrast, haplotype analysis in families with male offspring $(n=115)$ revealed a simulated overall significance corrected for multiple testing of $P=0.0076$, and as in the complete sample, the smallest uncorrected $P$-value of $P=0.0004$ was seen for marker combination rs363387and rs363333 (Table 5). 
Table 3 Single Marker P-Values for Alcohol- and Nicotine-Dependent Families as Well as for the Combined Family Sample

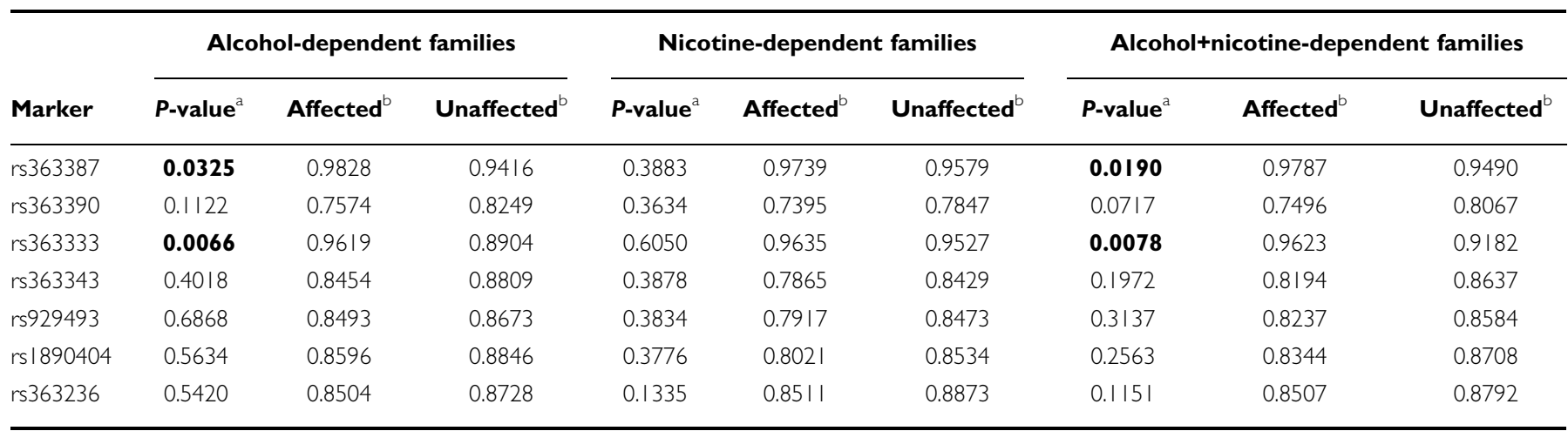

${ }^{\text {a }}$-values have been obtained using the FAMHAP program and are based on 10000 simulations. Bold values represent $P$-values $<0.05$.

'Frequency estimates for 'affected' are based on the frequency in a single affected offspring per family, frequency in 'unaffected' is calculated from the nontransmitted alleles to a single affected offspring per family; frequencies are given for the common allele.

Table 4 Single Marker P-values for the Subsample of AlcoholDependent Families $(n=1 \mid 5)$ with Male Offspring

\begin{tabular}{|c|c|c|c|}
\hline Marker & $P$-value ${ }^{a}$ & Affected $^{b}$ & Unaffected $^{\mathrm{b}}$ \\
\hline rs363387 & 0.0011 & 0.9950 & 0.9370 \\
\hline Rs363390 & 0.0402 & 0.7378 & 0.8291 \\
\hline rs363333 & 0.0035 & 0.9682 & 0.8925 \\
\hline rs363343 & 0.5629 & 0.8393 & 0.8710 \\
\hline rs929493 & 0.7748 & 0.8462 & 0.8638 \\
\hline rs 1890404 & 0.6565 & 0.8573 & 0.8808 \\
\hline rs363236 & 0.5234 & 0.8486 & 0.8785 \\
\hline \multicolumn{4}{|c|}{$\begin{array}{l}\text { a } P \text {-values have been obtained using the FAMHAP program and are based on } \\
\text { I } 0000 \text { simulations. Bold values represent } P \text {-values }<0.05 \text {. } \\
\text { 'Frequency estimates for 'affected' are based on the frequency in a single } \\
\text { affected offspring per family, frequency in 'unaffected' is calculated from the } \\
\text { nontransmitted alleles to a single affected offspring per family; frequencies are } \\
\text { given for the common allele. }\end{array}$} \\
\hline
\end{tabular}

No statistical significant $P$-values were observed in sexstratified samples of nicotine-dependent families. The male only combined sample ( $n=167$ families) revealed a global $P$-value of 0.018 suggesting that gender-specific effects might not be present in the nicotine-dependent sample.

\section{DISCUSSION}

We have analyzed seven DNA sequence variants located in the genomic region of the SLC18A2 gene on chromosome 10q26.11 for association with alcohol and nicotine dependence using a family-based design. Two single SNPs as well as several haplotypes revealed statistical significant association with alcohol dependence. Combining alcohol- and nicotine-dependent families again revealed association with the same two SNPs. None of the analyzed SNPs are known to cause functional changes of the protein encoded by the SLC18A2 gene. Since mice lacking the neuronal isoform of the vesicular monoamine transporter do not survive for more than a few days (Uhl et al, 2000), quantitative changes

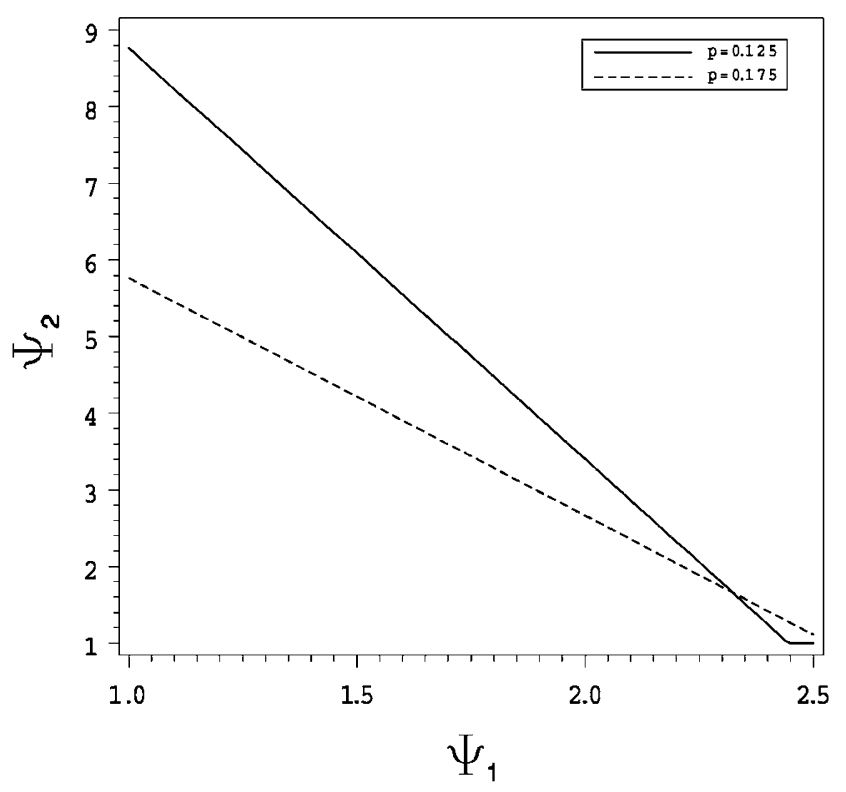

Figure I Relative risks $\left(\Psi_{1}, \Psi_{2}\right)$ resulting in a power of $80 \%$ for the sample of 131 alcohol-dependent families $(\alpha=0.05)$ and different population allele frequencies. For points $\left(\Psi_{1}, \Psi_{2}\right)$ above the line (either $P=0.125$ or 0.175$)$ the power is $>0.80 \%$.

in function of SLC18A2 are more likely to be involved in drug dependence. Interestingly, one of the analyzed SNPs, rs363387, which is associated with alcohol and drug dependence in our family samples as a single marker and as a component of associated haplotypes is located in exon 2 of the gene and results in a silent mutation (T68T). As the transversion $T \rightarrow G$ affects the third base of a codon, a strong effect of this alteration on protein synthesis would not be expected. A more complete approach to resequence the coding region as well as the conserved noncoding regions of the gene will be needed to identify all possible variants and to study their impact on function.

Using rodents, gender differences in alcohol intake have been reported (Middaugh and Kelley, 1999; Middaugh et al, 
Table 5 Details of Haplotype Analysis for Three-Marker Combinations Producing Smallest P-Values

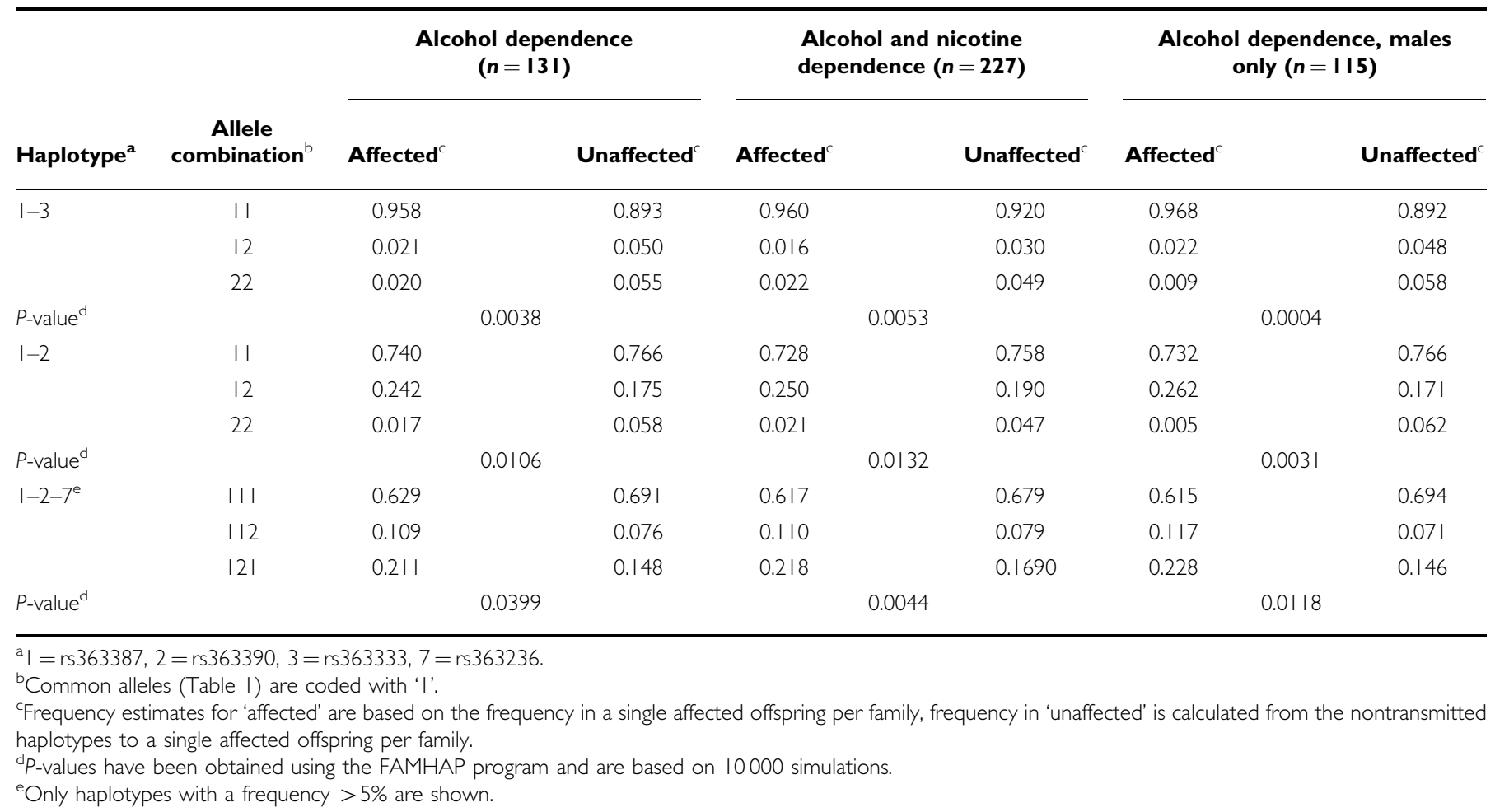

1999) with female animals frequently consuming more alcohol than male animals. Furthermore, Hall et al (2003) have studied sex-dependent modulation of ethanol consumption in SLC18A2 heterozygous knockout mice. They were able to show that male SLC18A2 heterozygous knockout mice consumed larger amounts of higher concentrations of ethanol as compared to wild-type mice. This appears to be consistent with our finding of male alcohol-dependent individuals mainly contributing to the observed association. However, we would like to point out that due to the small sample size of female alcoholdependent individuals we do not like to conclude that our finding is limited to male alcoholics only. Further investigation with a larger female alcohol dependent sample is warranted.

There is substantial evidence that individuals susceptible to dependence or addiction tend to be multiple substance abusers (Hiroi and Agatsuma, 2004). Therefore, we have combined the samples with nicotine- and alcohol dependence for analysis. $P$-values for single-marker analysis were approximately the same in the combined sample as for the sample with alcohol dependence only, suggesting a contribution of the sample with nicotine-dependent families to the association finding. However, haplotype analysis revealed marker combinations with an intermarker distance of about $35 \mathrm{~kb}$ still being associated with addiction in the combined sample. For the sample of alcohol dependence, associated haplotypes span only approximately $15 \mathrm{~kb}$. This might indicate that different variants in the $3^{\prime}$ and $5^{\prime}$ region of the gene are important if drug addiction as a general risk factor is regarded in contrast to alcohol dependence by itself. Since we were not able to establish evidence for statistical significant association using the nicotine-dependent sample alone, further investigation addressing this possibility is definitely needed.

To our knowledge, this is the first report of association of polymorphisms in the genomic region of the SLC18A2 gene with alcoholism or substance dependence. We have chosen a family-based approach in order to avoid confounding factors like population stratification. In addition, the family-based design is helpful in assigning haplotypes, and reduces genotyping errors by providing a check for Mendelian inheritance. In order to test if the alcohol-dependent sample had enough power to detect association for the nonassociated SNPs (rs363343, rs929493, rs1890404, rs363236), we performed power calculations. As can be seen from Figure 1, our sample of 131 alcoholdependent families had a power of $>80 \%$ to detect an association $(\alpha=0.05)$ provided that the relative risks are $\Psi_{1}=2$ and $\Psi_{2}=4$ and that the population frequency of the associated allele is 0.125 or 0.175 . A limitation of our study might be the number of tests being performed for the analysis of different phenotypes, subgroups, and marker combinations. In this context, we would like to point out that the global $P$-values, calculated by FAMHAP is a $P$-value which is adjusted for the number of different marker combinations, but is not corrected for the multiple testing issue induced by considering more than one phenotype and by performing subgroup analysis. Until replication in additional samples has been shown or, a functional relevance of one of the analyzed SNPs for substance dependence is demonstrated, this finding should be considered as preliminary. 


\section{ACKNOWLEDGEMENTS}

We are indebted to the subjects who volunteered for this study. We gratefully acknowledge the help in recruiting families by Eva Sohne. This work was supported by a grant from the Nationales Genomforschungsnetz (NGFN), Bundesministerium fuer Bildung und Forschung (BMBF), Germany to SGS, DBW and PEF.

\section{REFERENCES}

Becker T, Knapp M (2004a). Maximum-likelihood estimation of haplotype frequencies in nuclear families. Genet Epidemiol 27: 21-32.

Becker T, Knapp M (2004b). A powerful strategy to account for multiple testing in the context of haplotype analysis. Am J Hum Genet 75: 561-570.

Bierut LJ, Rice JP, Goate A, Hinrichs AL, Saccone NL, Foroud T et al (2004). A genomic scan for habitual smoking in families of alcoholics: common and specific genetic factors in substance dependence. Am J Med Genet A 124: 19-27.

Cloninger CR, Sigvardsson S, Gilligan SB, von Knorring AL, Reich T, Bohman M (1988). Genetic heterogeneity and the classification of alcoholism. Adv Alcohol Subst Abuse 7: 3-16.

Dinwiddie SH, Reich T (1993). Genetic and family studies in psychiatric illness and alcohol and drug dependence. J Addict Dis 12: 17-27.

Franke P, Schwab SG, Knapp M, Gansicke M, Delmo C, Zill P et al (1999). DAT1 gene polymorphism in alcoholism: a family-based association study. Biol Psychiatry 45: 652-654.

Hall FS, Sora I, Uhl GR (2003). Sex-dependent modulation of ethanol consumption in vesicular monoamine transporter 2 (VMAT2) and dopamine transporter (DAT) knockout mice. Neuropsychopharmacology 28: 620-628.

Heatherton TF, Kozlowski LT, Frecker RC, Fagerstrom KO (1991). The Fagerstrom Test for nicotine dependence: a revision of the Fagerstrom Tolerance Questionnaire. Br J Addict 86: 1119-1127.

Heinz A, Siessmeier T, Wrase J, Hermann D, Klein S, GrusserSinopoli SM et al (2004). Correlation between dopamine $\mathrm{D}(2)$ receptors in the ventral striatum and central processing of alcohol cues and craving. Am J Psychiatry 161: 1783-1789.

Henry JP, Botton D, Sagne C, Isambert MF, Desnos C, Blanchard V et al (1994). Biochemistry and molecular biology of the vesicular monoamine transporter from chromaffin granules. J Exp Biol 196: $251-262$.
Hiroi N, Agatsuma S (2004). Genetic susceptibility to substance dependence. Mol Psychiatry print copy in press (originally published online December 7, 2004, at www.nature.com/mp/ journal/vaop/ncurrent/.

Johnson Jr RG (1988). Accumulation of biological amines into chromaffin granules: a model for hormone and neurotransmitter transport. Physiol Rev 68: 232-307.

Knapp M (1999). A note on power approximations for the transmission/disequilibrium test. Am J Hum Genet 64: 1177-1185.

Knapp M, Becker T (2003). Family-based association analysis with tightly linked markers. Hum Hered 56: 2-9.

Madden PA, Heath AC, Starmer GA, Whitfield JB, Martin NG (1995). Alcohol sensitivity and smoking history in men and women. Alcohol Clin Exp Res 19: 1111-1120.

Middaugh LD, Kelley BM (1999). Operant ethanol reward in C57BL/6 mice: influence of gender and procedural variables. Alcohol 17: 185-194.

Middaugh LD, Kelley BM, Bandy AL, McGroarty KK (1999). Ethanol consumption by C57BL/6 mice: influence of gender and procedural variables. Alcohol 17: 175-183.

Robinson TE, Berridge KC (2003). Addiction. Annu Rev Psychol 54: $25-53$.

Sigvardsson S, Bohman M, Cloninger CR (1996). Replication of the Stockholm Adoption Study of alcoholism. Confirmatory crossfostering analysis. Arch Gen Psychiatry 53: 681-687.

Straub RE, Sullivan PF, Ma Y, Myakishev MV, Harris-Kerr C, Wormley B et al (1999). Susceptibility genes for nicotine dependence: a genome scan and followup in an independent sample suggest that regions on chromosomes $2,4,10,16,17$ and 18 merit further study. Mol Psychiatry 4: 129-144.

Sullivan PF, Kendler KS (1999). The genetic epidemiology of smoking. Nicotine Tob Res 1(Suppl 2): S51-S57; discussion S69-S70.

Sullivan PF, Neale BM, van den Oord E, Miles MF, Neale MC, Bulik $\mathrm{CM}$ et al (2004). Candidate genes for nicotine dependence via linkage, epistasis, and bioinformatics. Am J Med Genet B Neuropsychiatr Genet 126: 23-36.

True WR, Xian H, Scherrer JF, Madden PA, Bucholz KK, Heath AC et al (1999). Common genetic vulnerability for nicotine and alcohol dependence in men. Arch Gen Psychiatry 56: 655-661.

Uhl GR, Li S, Takahashi N, Itokawa K, Lin Z, Hazama M et al (2000). The VMAT2 gene in mice and humans: amphetamine responses, locomotion, cardiac arrhythmias, aging, and vulnerability to dopaminergic toxins. FASEB J 14: 2459-2465. 